\title{
PROPOSTA DE INDICADOR ESTRATÉGICO SIMPLIFICADO PARA CENTROS DE DISTRIBUIÇÃO DA MARINHA DO BRASIL
}

\author{
Guilherme Penha Pinto ${ }^{1}$ \\ Marinha do Brasil \\ guilherme.penha.pinto@gmail.com \\ Roberto Nogueira Marques ${ }^{2}$ \\ Marinha do Brasil \\ economia_roberto@yahoo.com.br
}

Resumo

Na Marinha do Brasil os diversos Centros de Distribuição prestam serviços semelhantes com distintos resultados de eficiência, eficácia e efetividade. Foi possível averiguar estatisticamente que a localização afeta o desempenho das atividades logísticas. Assim sendo este trabalho propõe uma modelagem econométrica para avaliar as atividades internas, atuando como um painel de indicadores, para avaliação, atenuação dos fatores de localização e auxílio para alocação de recursos escassos do setor de Abastecimento, por meio do benchmarking institucional. Enfim, o Sistema Gerencial necessita alterar o indicador atendendo aos anseios de um cenário calcado na economia da estratégia com uma visão bem definida.

Palavras-Chave: Logística, Modelagem e Abastecimento.

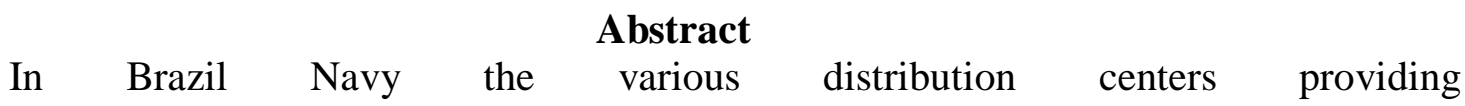
similar services with different results in efficiency, efficacy and effectiveness. It was possible to statistically determine the location affects performance of logistics activities. Therefore this paper proposes an econometric modeling to assess the internal activities, acting as a dashboard of indicators for assessment, mitigation and relief location factors for allocation of scarce resources of the supply sector through institutional benchmarking. Anyway, the Management System needs to change the indicator given the yearnings of a trampled in the strategy with a well-defined vision economy scenario.

Keywords: Logistics and Supply Modeling.

\section{Introdução}

\footnotetext{
${ }^{1}$ Oficial do Quadro Complementar de Intendentes de Marinha. Mestre em Economia Aplicada pelo PPGOM\UFPel. Lattes: http://lattes.cnpq.br/5364216817204672.

${ }^{2}$ Oficial do Quadro Complementar de Intendentes de Marinha. Lattes: http://lattes.cnpq.br/5364216817204672.
} 
O fenômeno da interdependência entre as instituições em nível global, o cenário de alta competição dos mercados, a multiplicidade e a velocidade das evoluções no arcabouço do conhecimento tácito das organizações, e a constante assimetria de informações requerem instituições públicas com soluções originais para o enfrentamento dos desafios do cotidiano. Neste ambiente em que se inserem os Centros de Distribuição (CD) da Marinha do Brasil (MB) solicita destes uma postura calcada na economia da estratégica. Assim sendo, diluem-se os espaços para atitudes efêmeras sem resultado de longo prazo. A confiança do cliente é fator necessário para o sucesso de um CD inserido em uma Cadeia de Suprimentos (CS) suscetível de entrada de novos agentes em um mercado competitivo.

Segundo Souza, Schnorr e Ferreira (2013) as alterações no ambiente funcional dos agentes, as ações empreendidas em função da globalização e os aperfeiçoamentos da tecnologia trouxeram a necessidade da atualização dos processos por meio da gestão estratégica. Os mesmos autores vislumbraram que focando na logística e na gestão da cadeia de suprimentos, poder-se-ia incrementar a eficiência e eficácia da organização.

Conforme Paiva Jr. (2010) a Gestão Estratégica, consolidada no setor privado, está sendo incorporada, paulatinamente, à Administração Pública. Pode-se com um procedimento de tomada de decisões, e mais além, por meio da consolidação das rotinas de decisão, com a implantação de ações para sua concepção, desenvolvimento, implementação e sustentação da estratégica, implementar e consolidar uma vantagem competitiva.

Na MB os Centros de Intendência da Marinha (CeIM) são a faceta do CD regional, cuja missão, resumidamente, é de representante do Sistema de Abastecimento da Marinha (SAbM) na sua área de jurisdição. Além disso, atuam como executor das atividades de obtenção, Unidade Gestora Executora (UGE) dos créditos orçamentários e Organização Centralizadora (OC) de pagamento de pessoal.

Engajada no aperfeiçoamento das atividades das diversas CS a MB instituiu, conforme Brasil (2011a), por meio da NORMAB-20-01, o Prêmio de Eficiência CeIM que está imbuído do propósito de atribuir critérios para a avaliação comparativa entre CeIM que possuem as atribuições acima destacadas. Porém, apesar da denominação, avalia apenas o aspecto de representante do SAbM, necessitando a inclusão de índices para as demais tarefas.

O presente trabalho visa sugerir o aperfeiçoamento das técnicas de comparação entre os CeIM, especificamente, quanto ao desempenho como CD regionais, focando-se nas atividades do SAbM, portanto sugerindo a alteração da denominação. Para tanto, demonstram-se, nas seções a seguir, índice alternativo para análise de eficiência, considerando o foco na atividade funcional, as especificidades regionais e o recompletamento de estoques executado pelo Órgão Gerencial, oferecendo aos gestores uma ferramenta de acompanhamento da evolução do SAbM para a agregação de valor enfrentando os concorrentes privados. Além dessa introdução, na seção 2 tem-se uma análise teórica, na seção 3 os procedimentos metodológicos, em seguida os resultados e finalizando uma breve conclusão.

\section{Fundamentação Teórica}

No referencial teórico, busca-se trazer o entendimento sobre a integração entre o referencial teórico e o processo de administração da CS.

\subsection{Logística e Cadeia de Suprimentos na Marinha do Brasil}

Conforme Paiva Jr. (2010), o conceito funcional de logística na MB foi influenciado por autores como Carl Phillip Gottlieb von Clausewitz (1780-1831), Antoine-Henri Jomini (1779-1869), sendo que o Manual de Logística da MB cita diretamente estes autores militares, sendo a atividade de abastecimento um processo logístico fadado a encaminhar os suprimentos desde a jusante, fornecedores, até a montante, cliente final. Criando-se para isso 
um sistema o Sistema de Abastecimento da Marinha (SAbM) forjado a luz da Doutrina de Logística Militar estabelecida pelo Ministério da Defesa.

A Marinha do Brasil adota a seguinte definição para logística:

Logística é a componente da arte da guerra que tem como propósito obter e distribuir às Forças Armadas os recursos de pessoal, material e serviços em quantidade, qualidade, momento e lugar por elas determinados, satisfazendo as necessidades na preparação e na execução de suas operações exigidas pela guerra. (BRASIL, 200. p.45).

Conforme Morcillo (2008) conceitua-se o abastecimento no escopo da Marinha do Brasil:

\begin{abstract}
Abastecimento é um conjunto de atividades que tem o propósito de prever e prover, para as Forças e demais Organizações Militares da MB, o material necessário a mantê-las em condições de plena eficiência. Assim, o Abastecimento proporciona um fluxo adequado do material necessário, desde as fontes de obtenção até as OM Consumidoras (OMC), abrangendo a Função Logística Suprimento e parte da Função Logística Transporte, além de relacionar-se, estreitamente, com a Função Logística Manutenção (MORCILLO, 2008, p.26).
\end{abstract}

Como se pode depreender da nova Atitude Logística no Apoio a Sistemas Operativos (ATLAS), Brasil (2012), os novos desafios regrados pela Estratégia Nacional de Defesa, como a interoperabilidade, demandam da MB repensar o apoio Logístico calcado em Sistemas de Informação para apoio a decisão na visão de "Abastecer para Combater". O foco se volta para as Cadeias de Suprimento, tratadas como "Cadeias Logísticas de Abastecimento" por essa nova concepção de apoio logístico ATLAS. A gestão da cadeia de abastecimento comporá o Apoio Logístico Integrado (ALI) da MB, juntamente com a Catalogação e a Manutenção, tais funções não esgotam o ALI da MB, sendo citadas por serem competências necessárias ao SAbM.

\title{
2.2 CeIM como atores da Cadeia de Suprimentos
}

Para Santos (2006) os CD são indutores de diferenciação agregando valor pela disponibilidade dos itens da CS, criando soluções flexíveis para as necessidades customizando com agilidade frente às exigências dos clientes, oferecendo altos níveis de serviço ao cliente, por meio do baixo lead time (tempo de ressuprimento).

Conforme o Regulamento dos Centros de Intendência da Marinha, Brasil (2011b), a missão destas organizações militares é "contribuir para a prontidão dos meios navais, aeronavais e de fuzileiros navais, sediados ou em trânsito em suas respectivas áreas de responsabilidade, bem como dos estabelecimento de terra por eles apoiados" (BRASIL, 2011b, p. 1), esta definição demonstra, claramente, quais são os clientes dos CeIM. Assim sendo, por meio da Divisão de Abastecimento o CeIM atuará como CD atendendo os clientes da respectiva jurisdição.

Dentre os atributos de apoio logístico em CD apontados como relevantes, pela literatura acadêmica, os que aparecem com maior frequência no cotidiano são: disponibilidade de produto, tempo de ciclo do pedido, consistência do prazo de entrega, frequência das entregas, proporção do pedido atendido, informação de apoio, suporte técnico, qualidade da documentação, apoio na entrega física e merchandising.

Dos atributos apresentados, apenas um independe dos esforços de cada CeIM, qual seja a “disponibilidade de produto", que está diretamente ligada ao recompletamento de estoques pelos Órgãos de Distribuição (OD) primários administrados pelo agente de coordenação da cadeia de suprimentos, o Órgão Gerencial do SAbM, qual seja o Centro de Controle de Inventário da Marinha (CCIM), atuando como um monopsônio, uma vez que na 
citada cadeia é o único fornecedor do SAbM, sendo vedada a aquisição diretamente no comércio, exceto em condições extraordinárias. Ou seja, sem a disponibilidade de itens satisfeita, a missão dos CeIM, e seus respectivos serviços prestados, estão seriamente comprometidos.

\title{
2.3 A Economia da Estratégia e Tecnologia da Informação (TI)
}

Para Paiva Jr. (2010) a partir das informações do ambiente serão elaboradas de forma perene as estratégias que suportem os ganhos competitivos dos $\mathrm{CD}$, ainda segundo o autor:

\begin{abstract}
"O aprimoramento da logística interna das organizações, através da melhoria dos processos e do fluxo de dados e informações que trafegam em cada um dos departamentos e entre estas entidades, há tempos vem sendo objeto de preocupação. O monitoramento das atividades logísticas internas não é uma novidade. Evidentemente, dentro de uma organização existem vários processos logísticos. No entanto, o acompanhamento de indicadores para todos eles não é recomendado, sob pena de tornar o processo de coleta de dados demasiadamente complexo e dificultar a tomada de decisões diante de informações dispersas.” (PAIVA JR, 2010, p. 56).
\end{abstract}

Segundo Drucker apud Santos (2006), a logística é a última fronteira para a vantagem competitiva, portanto, o uso de CD permite conquistar uma posição única e sustentável, garantindo a participação em um mercado cada vez mais concorrido, exigente e atualizado com as necessidades de seus clientes, em especial os corporativos.

Ainda, segundo o mesmo autor, um "instrumento muito útil a ser utilizado na gestão estratégica é o benchmarking. Esta ferramenta permite às organizações avaliar a sua competitividade frente aos melhores do ramo de atividade. Competitividade esta, que deve estar associada às condições anteriores aos resultados obtidos" (DRUCKER apud SANTOS, 2006, p.10). O autor complementa, afirmando que na logística, são seguidos os mesmos passos para a criação da estratégia, abordagens inovadoras de estratégia de logística podem proporcionar vantagens competitivas, onde a escolha da melhor estratégia por sua vez, inclui três objetivos fundamentais: redução de custos, redução de capital e melhoria dos serviços.

Pode-se afirmar que "o sistema de apoio à decisão é um sistema de informação [...] que combina modelos e dados, em uma tentativa de solucionar problemas semi-estruturados com grande envolvimento por parte do usuário" (TURBAN, MCLEAN e WETHERBE, 2004, p. 12). Ainda, conforme Rainer Jr. e Cegielski (2011), a gerência estratégica dos processos focados no negócio da organização, devem ser apoiados por Sistemas de Informação que auxiliam em todos os aspectos da tomada de decisões complexas sobre um problema específico. Porém o SAbM carece de um algoritmo moderno de análise, como os apresentados no Figura 1, que provenha um indicador como parte da solução de governança logística, caracterizado segundo Gather Group (1998) como ferramentas de Business Inteligence (BI), no intuito de apoiar as atividade de Apoio Logístico Integrado.

\begin{tabular}{|l|l|}
\hline Tipo & Descrição \\
\hline $\begin{array}{l}\text { Business Inteligence } \\
\text { (Indicadores de desempenho) - } \\
\text { Estratégico }\end{array}$ & $\begin{array}{l}\text { Integra o sistema de inteligência da empresa, sendo monitorados } \\
\text { pelos diretores de Logística ou Supply Chain (Cadeia de } \\
\text { Suprimentos), possuindo no máximo } 5 \text { indicadores. }\end{array}$ \\
\hline $\begin{array}{l}\text { Key performance indicators - } \\
\text { KPIs - Tático }\end{array}$ & $\begin{array}{l}\text { Perfazem o acompanhamento diário ou semanal pelos gerentes de } \\
\text { Logística, representando de } 5 \text { a } 10 \text { indicadores mais relevantes. Parte } \\
\text { sendo utilizada na concepção do BI. }\end{array}$ \\
\hline $\begin{array}{l}\text { Métricas ordinárias - } \\
\text { Operacional }\end{array}$ & $\begin{array}{l}\text { Medidas de nível de supervisão para baixo. Monitoram o cotidiano da } \\
\text { operação. Viabilizam o processo bottom-up (de baixo para cima). } \\
\text { Proporcionam o envolvimento para soluções rápidas. }\end{array}$ \\
\hline
\end{tabular}

Figura 1 - Indicadores de Desempenho em Nível Estratégico 
Fonte: Neves (2009), Elaboração do autor.

O Sistema de Informações Gerenciais do Abastecimento (SINGRA) é definido nas normas sobre abastecimento:

É o sistema de informações e de gerência de material que se destina a apoiar as fases básicas das funções logísticas Suprimento, Transporte e Manutenção relacionadas ao Abastecimento, prevendo e provendo os recursos de informação (regras, informações e tecnologia) necessários ao desempenho das atividades técnicas e gerenciais de Abastecimento. (BRASIL, 2009).

O SAbM, por meio do SINGRA, trabalha com 22 diferentes indicadores, os quais podem ser visualizados no Figura 2, porém nenhum apresenta o dinamismo de um indicador para um painel de tomada de decisões logísticas.

\begin{tabular}{|c|c|c|}
\hline Nível & Definição & Indicador \\
\hline Estratégico & $\begin{array}{l}\text { Voltado para suporte às decisões de alto } \\
\text { nível e que venham a influenciar o } \\
\text { SAbM a longo prazo. }\end{array}$ & $\begin{array}{l}\text { - Nível de Serviço; } \\
\text { - Nível de Serviço de Projetos; } \\
\text { - Nível de Serviço Fardamento; } \\
\text { - Valor Contábil do Estoque; e } \\
\text { - Acurácia do Estoque. }\end{array}$ \\
\hline Tático & $\begin{array}{l}\text { Voltado para as decisões de nível } \\
\text { gerencial e que influenciam o SAbM a } \\
\text { médio prazo. }\end{array}$ & $\begin{array}{l}\text { - Giro de Estoque; } \\
\text { - Giro de Material; } \\
\text { - Tempo de Obtenção; } \\
\text { - Tempo de Atendimento de RM; } \\
\text { - Tempo de arrecadação; } \\
\text { - Tempo de Pagamento de Faturas; } \\
\text { - Itens com RM em Dívida; } \\
\text { - SE em aberto nas Comissões Navais no } \\
\text { Exterior; } \\
\text { - Ordens de Compra em Atraso; } \\
\text { - Itens Inventariados; } \\
\text { - Acurácia do Inventário; } \\
\text { - Índice de Arrecadação Parcial de RCL; } \\
\text { - Î́ndice de Pendências de Arrecadação de } \\
\text { RCL; e } \\
\text { - RCL sem registro de Nota Fiscal. }\end{array}$ \\
\hline Operacional & $\begin{array}{l}\text { Voltado para as decisões de nível } \\
\text { operacional. }\end{array}$ & $\begin{array}{l}\text { - Perícia do Material; } \\
\text { - Ajustes Contábeis; e } \\
\text { - Agregação de Pedidos de Obtenção a } \\
\text { Processo. }\end{array}$ \\
\hline
\end{tabular}

Figura 2 - Indicadores de Desempenho em Níveis por Importância

Fonte: Adaptado de Paiva Jr. (2010, p. 65).

\section{Procedimentos Metodológicos}

Conforme Gil (1999) a pesquisa desenvolvida neste trabalho é aplicada e exploratória, visando elucidar os mecanismos de comparação e acompanhamento das atividades funcionais dos CeIM, proporcionando aos gestores uma ferramenta de BI.

Para alcançar o objetivo exposto na Introdução, discute-se no presente seguimento os pressupostos e hipóteses e as linhas de ação para remodelagem do índice capacitando a ferramenta de TI do SAbM, calcado no documento normativo sobre abastecimento da MB: 
A eficácia de uma Força Naval repousa no grau de eficiência de seu setor de apoio logístico, o qual deve ser continuamente monitorado. Para tal, é imperioso que o SAbM possua mecanismos de controle que permitam a avaliação de efetivo desempenho dos seus órgãos, por meio de indicadores de desempenho que apresentem a performance real alcançada por ocasião da realização de determinado processo de Abastecimento. O subsistema de Controle do SINGRA deve ser utilizado como principal ferramenta de suporte ao controle dos diversos processos de Abastecimento executados pelos órgãos do SAbM, devendo estar plenamente capacitado a prover o apoio às consultas realizadas. (BRASIL, 2009, p.30)

Como pressupostos têm-se a acurácia na aferição dos parâmetros, por meio da disponibilidade de informações do Banco de Dados do Sistema de Gerenciamento do Abastecimento (BD-SINGRA) e como principal hipótese a admissão de disparidades regionais quanto a localização dos $\mathrm{CD}$. Portanto, quanto à coleta de dados, o BD-SINGRA apresenta uma consistente disponibilidade de dados estatísticos, com ampla confiança no registro, proporcionando a mensuração de vários aspectos intrínsecos do cotidiano dos CD.

Outro importante pressuposto é que as atividades internas dos CeIM, CD do SAbM, começam quando da análise dos pedidos dos clientes, Requisições de Material (RM), que possuam disponibilidade de estoque, ou seja, RM canceladas ou em dívida - aguardando recompletamento, não demonstram a eficácia dos procedimentos internos adotados pelo CD, ao qual compete analisar, segregar, separar, fornecer e efetuar os devidos registros de fornecimentos.

Conforme Gujarati (2000), para inferir qualquer comparação entre as amostras dos CeIM, supõe-se que os estimadores tendem para o valor verdadeiro, $\lim _{n \circledast \square} \square(\hat{\square})=\square$, a optação por parâmetros que competem à rotina dos CeIM (tempo de separação, segregação entre outros), e não aos parâmetros extra-CD, como tempo de recompletamento de estoques colaboram para a correta descrição da realidade.

Focando na avaliação pelas categorias de itens atendidos (Gerências de Suprimento), uma vez que nem todos os CeIM, estocam os mesmos itens, como por exemplo, medicamentos, neste caso, impossibilitando a comparação direta, abrangendo todos os itens de suprimento, entre os CD regionais. Porém é possível avaliar o desempenho por itens entre os CD regionais e compararem seus desempenhos com o CD nacional em uma avaliação marginal.

Na literatura, os estudos de caso requerem muito tempo para sua realização e, frequentemente, seus resultados não são consistentes. Porém, com a disponibilidade de dados, principalmente, quando avaliado a partir do histórico existente e, concomitante, às experiências acumuladas para períodos de curto prazo, como a atual sistemática, os resultados são passíveis de confirmação durante o tempo. Em vista o aperfeiçoamento do método associando medidas de eficiência aos benchmark extraídos dos casos observados no SAbM, demais CD, procura-se incentivar a adesão dos demais CeIM para a implementação de avaliações e outros mecanismos de coordenação e incentivo que utilizem a metodologia discutida neste seguimento.

Dado este cenário metodológico, uma inferência estatística, seria a comparação entre os CeIM, tratando os dados de tempo de atendimento de todos os CeIM como população finita e os dados de cada CeIM como uma amostra. Assim, uma vez que, os parâmetros populacionais são conhecidos, torna-se viável a mensuração e respectivos testes de hipótese e comparação de variâncias, ou seja, diferenças entre os CeIM e peculiaridades que auxiliaram na tomada de decisão pelo gerente do SAbM.

Os dados consolidados do BD-SINGRA contemplam dados para o SAbM, que incluem tempo de recompletamento, tal fato desconfigura o desvio padrão do tempo de atendimento dos CeIM, ou seja, as diferenças entre o tempo de atendimento ao cliente, em 
específico deturpa a avaliação das atividades de Controle, Armazenagem e Tráfego de Carga executadas pelos $C D$, uma vez que contempla no índice do nível de serviço o tempo de recompletamento. Tal problemática pode ser atenuada pela proposição de ajuste do algoritmo do referido nível.

Fazendo uso do indicador de Tempo de Atendimento do SINGRA, subtraído do tempo de recompletamento do estoque, segue sugestão para analisar gerencialmente os serviços prestados pelo centro de distribuição:

$$
\hat{Y}_{i}=\square_{0}+\square_{P I_{-} m a t 1} P I_{-} m a t_{1 i}+\square_{P I_{-} M_{a t}{ }_{i}} P I_{-} m t_{2 i}+\ldots .+\square_{P I_{-} M_{a t n}} P I_{-} m a t_{n i}+\square
$$

Sendo:

i) $\quad \hat{Y}_{i}$ : média de tempo de atendimento total do CD no mês i (um índice para comparação);

ii) $\quad \square_{0}$ : intercepto, o qual representa o tempo mínimo das atividades administrativas, passível de verificação;

iii) $\quad \square_{p i_{-} \text {mat }}$ : demonstra a sensibilidade do indicador ao item específico;

iv) PI_mat $t_{1 i}$ : tempo de atendimento médio das RM de um PI (Parte Identificadora do Item de Suprimento, ou simplesmente, código do Item) no mês i em dias;

v) $\quad$ i : $1,2 \ldots 12$; e

vi) PI_mat: Código identificador dos materiais atendidos naquele mês.

Na Equação 1, cada item, terá seu tempo de atendimento mensurado conforme o método do indicador do SINGRA. Com a estimação do tempo médio total Y e a estimação de cada item de suprimento, o objetivo será alcançar a minimização do tempo total médio de atendimento do $\mathrm{CD}$, não sendo sugerido no presente trabalho quaisquer restrições. Tal ferramenta possibilitará a análise tempo de atendimento entre os itens, possibilitando um benchmark com outros agentes individualizados do SAbM e extra-MB, ou ainda, com a média populacional do Sistema de Abastecimento.

A regressão da Equação 1 possibilitará, também, a comparação da sensibilidade do tempo de atendimento de cada Item de Suprimento $\left(D_{P I_{-} m a t}\right)$, ou seja, quanto um determinado item está contribuindo para o incremento no tempo de atendimento total do CD. Esta última informação, por itens, poderá subsidiar decisões de alocação de recursos em material e adestramento para a melhoria de processos de separação e expedição deficientes e estudar os métodos, relativamente, mais eficientes para que se tornem benchmarks.

Além dos dados comportados pela Equação 1, poder-se-ia analisar uma variável dummy (0 ou 1), para mensurar a sensibilidade quanto à localização do CeIM em uma capital (0) ou distante de grandes centros (1), seria de fácil aplicação e poderia ser avaliada por testes de significância, tendo como hipóteses que a localização do CeIM em uma capital diminui o tempo de atendimento em função da disponibilidade de serviços em especial tráfego de carga, a variável dummy executaria a mesma lógica econômica de mensuração, por exemplo, de uma variável proxy do nível de desenvolvimento da cidade, qual seja o PIB (Produto Interno Bruto) da cidade ou região microrregião onde está instalado o CeIM. 


\section{Resultados}

Este seguimento expõe os resultados econométricos referentes à estimação da equação, referida na metodologia, a qual é a representação da modelagem a ser estudada. $\mathrm{O}$ modelo determina a relação entre a variável dependente - média do tempo de atendimento, e as variáveis explicativas do modelo - tempo de atendimento dos itens de suprimento. Ainda, realiza-se a análise das hipóteses apresentadas na seção anterior, criando um paralelo destas suposições com os resultados obtidos na regressão econométrica.

O modelo tem seus resultados apresentados nas Tabelas a seguir. Como exposto no capítulo anterior, $Y_{i}$ é a variável dependente, a qual reflete o tempo de atendimento média total de todos os CD. Virtude a sensibilidade dos dados estratégicos de suprimento na MB, optouse por analisar apenas as gerências de material comum e gêneros alimentícios, para aferir o modelo e suas hipóteses, e além disso foi utilizada média da gerência para não expor a sensibilidade de um item específico.

Uma das hipóteses aventadas é de que a localização pode afetar o tempo de atendimento em função das melhores condições de infra-estrutura. Para verificar essa situação utilizou-se uma amostra em uma regressão dicotômica tipo logit para verificar da prudência da inserção de uma variável dicotômica independente na regressão da Equação 1. Comporam a amostra os CeIM (CD) de Salvador-BA, Belém-PA, Ladário-MS, Manaus-MA, Rio Grande - RS e Natal-RN. Na averiguação a seguir, a localização é uma variável dependente dicotômica, de uma regressão tipo logit, sendo 0 para Salvador\Belém\Natal e 1 para outra cidade, vale registrar que Manaus em virtude das peculiaridades de acesso não foi tratada como grande centro facilitador de modais logísticos. Os resultados demonstram que é consistente a hipótese de que a localização em uma metrópole com bom acesso logístico enviesa os dados da regressão da Equação 1, sendo necessária a inserção da variável dicotômica. Porém, cabe ressaltar que o volume de pedidos não é preponderante quanto a localização, mas sim o tempo de atendimento como esperado.

\begin{tabular}{lc} 
Tabela 1 - Análise de Salvador e Belém - JAN 2011 - DEZ 2012 \\
\hline Variável & Coeficientes \\
\hline Pedidos Gêneros & $-0,000511$ \\
& $(-0,4801)$ \\
Pedidos de Material Comum & 0,002948 \\
& $(1,386)$ \\
Tempo de Atendimento & 0,025604 \\
& $(2,523)^{*}$ \\
\hline Observações & 169 \\
$R^{2}$ de McFadden & 0,089685 \\
Log likelihood & $-91,49511$ \\
Qui-quadrado(3) & 18,0284 \\
& $(p=0,0004)^{*}$ \\
\hline
\end{tabular}

Fonte: Cálculos do Autor

Nota: As estatísticas z são apresentadas em parênteses para as variáveis explicativas.

* variável significativa a $5 \%$.

Em vista os resultados da Tabela 1, que demonstram que o tempo de atendimento está fortemente relacionado com a localização, insere-se no modelo advindo da Equação 1 e uma variável independente dicotômica, qual seja 0 para Salvador\Belém $\backslash N a t a l$ e 1 para outra cidade. A Tabela 2 demonstra estes resultados, sendo esperado um aumento de aproximadamente 23 dias para os CD que não se localizam em Salvador e Belém. Portanto, na Tabela 2, demonstra-se preliminarmente a aferição de qual gerencia seria mais sensível, para 
todos os CeIM, podendo-se verificar que itens de material comum e gêneros afetam em maior grau o tempo de atendimento.

Tabela 2 - Regressão do modelo por gerenciais JAN2011 - DEZ 2012

\begin{tabular}{lc}
\hline Variável & Coeficientes \\
\hline Tempo médio de Gêneros & 0,50287 \\
Tempo médio de Material Comum & $(2,7920)$ \\
& 0,329414 \\
Dummy Salvador|Belém & $(14,9084)^{*}$ \\
& 5,79712 \\
Observações & $(4,1316)^{*}$ \\
$R^{2}$ & 169 \\
& 0,835891 \\
\hline Log likelihood & \\
Teste de Breusch-Pagan & $-630,0174$ \\
& 236,627 \\
Estatística F & $(p=4,14019 \mathrm{e}-052)^{*}$ \\
& 280,1426 \\
\end{tabular}

Fonte: Cálculos do Autor

Nota: As estatísticas $t$ são apresentadas em parênteses para as variáveis explicativas.

* variável significativa a $1 \%$.

Os dados revelam que o tempo de Gêneros não é preponderante para o tempo de atendimento, ou seja, a atividade operacional interna do CD não afeta o tempo de atendimento. Porém, o tempo de Material comum está significativamente influenciando o tempo total de atendimento dos CD. Em especial a variável de localização dummy para Salvador \Belém\Natal revela que se o CD não está localizado em um destes grandes Centros urbanos existe incremento significativo no tempo médio de atendimento (o antilog (dummy) $\equiv$ $\infty)$.

Reforçando a questão da localização do CD, torna-se imperativo analisar especificamente como o tempo dos procedimentos internos estão relacionados entre metrópole e interior. As Tabelas 3 e 4 demonstra-se como o tempo de liberação e fornecimento de pedidos se comportam em Salvador e Ladário afetando o tempo total da gerencia.

Os testes demonstram o esperado para identidades que possuem o objetivo de demonstram relações de influencia. Os resultados revelam que Salvador possui processos internos das citadas gerenciais, relativamente, influenciando em menor grau a média do tempo de atendimento total do CD. Apesar das diferenças serem tênues, corroboram a hipótese que um grande centro possui melhores condições para o desenvolvimento da atividade logísticas apesar dos aparentes esforços do CD do interior, para o caso estudado.

A Tabela 5, Apêndice, revela em termos de comparação que as médias entre um CD de uma grande metrópole (Salvador) e um CD do interior (Ladário) possuem grande diferença no tempo de atendimento, liberação e fornecimento, sendo que todas as médias da metrópole superam o interior, reiterando o que foi revelado pelas Tabelas 3 e 4 e por meio da variável dummy na Tabela 2. 
Tabela 3 - Identidade de Ladário por gerenciais - JAN 2005 - DEZ 2012

(continua)

\begin{tabular}{lcc}
\hline Variável & Coeficientes \\
\hline Liberação de Gêneros & 1,3466 \\
& $(10,75)^{*}$ & 0,8989 \\
Fornecimento de Gêneros & $(11,28)^{*}$ \\
Tabela 3 - Identidade de Ladário por gerenciais - JAN 2005 - DEZ 2012 & (conclusão) \\
Liberação de Material Comum & 0,9607 \\
& $(146,8)^{*}$ \\
Fornecimento de Material Comum & 0,9001 \\
& $(52,95)^{*}$ \\
\hline Observações & 96 \\
\hline$R^{2}$ & 0,9988 \\
Log likelihood & $-238,0174$ \\
Teste de Breusch-Pagan & 32,627 \\
Estatística F & $(p=4,78 \mathrm{e}-07)^{*}$ \\
& 20676 \\
\hline
\end{tabular}

Fonte: Cálculos do Autor

Nota: As estatísticas $t$ são apresentadas em parênteses para as variáveis explicativas.

* variável significativa a $1 \%$.

\begin{tabular}{lc} 
Tabela 4 - $\quad$ Identidade de salvador por gerenciais & JAN 2005 - DEZ 2012 \\
\hline Variável & Coeficientes \\
\hline Liberação de Gêneros & 1,1733 \\
& $(3,47)^{*}$ \\
Fornecimento de Gêneros & 0,8639 \\
& $(7,07)^{*}$ \\
Liberação de Material Comum & 1,0066 \\
& $(99,30)^{*}$ \\
Fornecimento de Material Comum & 0,8083 \\
& $(43,48)^{*}$ \\
\hline Observações & 966 \\
$R^{2}$ & 0,9982 \\
Log likelihood & $-225,5927$ \\
Teste de Breusch-Pagan & 60,4278 \\
& $(p=4,7 e-13)^{*}$ \\
Estatística F & 12972 \\
& $(p=1 \mathrm{e}-125)^{*}$ \\
\hline
\end{tabular}

Fonte: Cálculos do Autor

Nota: As estatísticas t são apresentadas em parênteses para as variáveis explicativas.

* variável significativa a $1 \%$.

\section{Conclusão}

A relevância desse estudo está relacionada à importância dessa ferramenta estratégica no cenário competitivo, como instrumento de suporte à gestão organizacional. A construção argumentativa foi baseada em um estudo descritivo e quantitativo, por meio de análise crítica, obtendo-se uma apreciação conclusiva sobre a importância do foco pelas partes relacionadas, por meio da compatibilidade e sinergia da aferição de desempenho nas organizações.

A pesquisa amplia o conhecimento sobre a aplicação dos indicadores de desempenho, adotados no setor público, sugerindo complementações e adoções de novas perspectivas aos 
modelos vigentes na MB. O presente estudo fornece um diagnóstico sobre a metodologia, compatível às necessidades atuais do mundo corporativo, assim como detalha melhores práticas para uma adoção ótima dos processos com foco na Excelência em Gestão.

A análise da eficiência é condição para o aperfeiçoamento das atividades dos CeIM, visa-se a acurácia na aferição, oportunizando o planejamento das inversões de recursos nos processos, relativamente, desatualizados para o atendimento dos meios navais, de fuzileiros navais e aeronavais. Portanto, a modelagem focada no grau de eficiência do setor de Abastecimento dos CeIM, evita fragmentação do problema de pesquisa que pode tomar um rumo de generalização com aspectos negativos para o alcance de soluções por meio do benchmarking corporativo, sugerindo-se a alteração da nomenclatura da NORMAB-20-01, porém mantendo o foco no Abastecimento e aperfeiçoando o algoritmo de mensuração de desempenho, preparando os CD da MB para o enfrentamento das ameaças da concorrência privada por meio da agregação de valor ao cliente, principalmente, menor tempo de atendimento.

\section{Referências Bibliográficas}

BRASIL. Estado Maior da Armada. Manual de Logística da Marinha: EMA-400. 2. rev. Brasília, DF, 2003.

Secretaria Geral da Marinha. Normas para Execução do Abastecimento: SGM201. 6. rev. v. 1. Rio de Janeiro, RJ, 2009.

Secretaria Geral da Marinha. Normas do Abastecimento: Prêmio de Eficiência do

CeIM. Rio de Janeiro, RJ, 2011a.

. Comando de Operações Navias. Portaria $n^{\circ} 43$ de 2011. Aprova o Regulamento dos

Centros de Intendência da Marinha. Rio de Janeiro, RJ, 2011b.

. Secretaria Geral da Marinha. III Jornada de Interoperabilidade Logísticag: Nova

Atitude Logística no Apoio a Sistemas Operativos. Rio de Janeiro, RJ, 2012. Palestra ministrada aos militares das forças armadas do Brasil e convidados.

GIL, A. C. Métodos e técnicas de pesquisa social. 5a edição. São Paulo: Atlas, 1999.

GUJARATI, D. N. Econometria Básica. 3 ed. São Paulo: Makron Books, 2000.

MORCILLO, R. Um estudo sobre a adequabilidade dos critérios de seleção para estoque pelo sistema de abastecimento da Marinha do Brasil dos itens de material de saúde com base na criticalidade e no custo de armazenagem. Monografia (Curso de Aperfeiçoamento Avançado em Administração e Logística de Material). Rio de Janeiro: Centro de Instrução Almirante Wandenkolk, 2008.

NEVES, M. A. O. Tudo sobre indicadores de desempenho em logística. Revista Mundo Logística, Curitiba, n. 12, p. 30 - 45, 2009.

PAIVA JR., C. S. Gestão estratégica do abastecimento do ponto de vista da logística integrada: proposta de um framework de alinhamento com a estratégica da Diretoria de Abastecimento da Marinha. 2010. 91f. Trabalho de Conclusão de Curso (Curso de Aperfeiçoamento Avançado em Administração e Logística de Materiais) - Faculdade de Administração e Finanças - UERJ, Rio de Janeiro, 2010.

RAINER JR., R. K.; CEGIELSKI, C. G. Introdução a Sistemas de Informação: apoiando e transformando negócios na era da mobilidade. 3 ed. Rio de Janeiro: Elsevier, 2011.

SANTOS, A. Centros de distribuição como vantagem competitiva. Revista de Ciências Gerenciais, São Paulo, v. 10, n. 12, p 34-40, 2006.

SOUZA, A. M.; SCHNORR. C.; FERREIRA, B.F. Práticas de gestão de custos logísticos: Estudo de caso de um empresa do setor alimentício. Revista Contemporânea de Contabilidade. Florianópolis, v. 10, n.19. p.03-32. JAN\ABR. 2013.

TURBAN, E.; MCLEAN, E.; WETHERBE, J. C. Tecnologia da Informação para Gestão. 3. ed. Porto Alegre: Bookman, 2004. 


\section{Apêndice}

Tabela 5 - Indicadores dos CeIM de Salvador e Ladário

\begin{tabular}{r|rrr|r|r|r}
\hline \multirow{2}{*}{} & \multicolumn{7}{c}{ Gêneros } \\
\cline { 2 - 7 } & \multicolumn{7}{|c|}{ Salvador } & \multicolumn{3}{c}{ Ladário } \\
\cline { 2 - 7 } & $\begin{array}{c}\text { Tempo } \\
\text { Médio de } \\
\text { Período }\end{array}$ & $\begin{array}{c}\text { Tempo de } \\
\text { Atendimento } \\
\text { Liberação }\end{array}$ & $\begin{array}{c}\text { Tempo de } \\
\text { Fornecimento } \\
\text { Médio de } \\
\text { Atendimento }\end{array}$ & $\begin{array}{c}\text { Tempo de } \\
\text { Liberação }\end{array}$ & $\begin{array}{c}\text { Tempo de } \\
\text { Fornecimento }\end{array}$ \\
\hline 2012 & 3,39 & 1,22 & 2,54 & 3,41 & 1,12 & 4,17 \\
2011 & 4,61 & 2,08 & 3,50 & 2,77 & 1,33 & 3,15 \\
2009 & 3,35 & 0,48 & 4,37 & 5,47 & 1,64 & 5,41 \\
2008 & 3,69 & 0,56 & 4,60 & 3,42 & 1,83 & 2,22 \\
2007 & 4,33 & 0,81 & 4,58 & 6,57 & 2,14 & 5,83 \\
2006 & 3,60 & 0,81 & 3,63 & 8,19 & 1,35 & 7,69 \\
2005 & 2,11 & 0,66 & 1,72 & 3,62 & 1,13 & 2,63 \\
\hline Média: & 2,37 & 0,46 & 2,24 & 5,66 & 3,92 & 2,06 \\
\hline
\end{tabular}

\begin{tabular}{|c|c|c|c|c|c|c|}
\hline \multirow[b]{3}{*}{ Período } & \multicolumn{6}{|c|}{ Material Comum } \\
\hline & \multicolumn{3}{|c|}{ Salvador } & \multicolumn{3}{|c|}{ Ladário } \\
\hline & $\begin{array}{c}\text { Tempo } \\
\text { Médio de } \\
\text { Atendimento }\end{array}$ & $\begin{array}{l}\text { Tempo de } \\
\text { Liberação }\end{array}$ & $\begin{array}{c}\text { Tempo de } \\
\text { Fornecimento }\end{array}$ & \begin{tabular}{|c|} 
Tempo \\
Médio de \\
Atendimento
\end{tabular} & $\begin{array}{l}\text { Tempo de } \\
\text { Liberação }\end{array}$ & $\begin{array}{c}\text { Tempo de } \\
\text { Fornecimento }\end{array}$ \\
\hline 2012 & 34,35 & 18,54 & 18,39 & 49,64 & 27,78 & 25,10 \\
\hline 2011 & 46,90 & 20,72 & 33,40 & 46,43 & 35,05 & 13,75 \\
\hline 2010 & 66,34 & 45,98 & 23,52 & 130,22 & 104,32 & 33,63 \\
\hline 2009 & 64,54 & 49,06 & 16,93 & 106,79 & 80,09 & 30,59 \\
\hline 2008 & 55,64 & 41,60 & 15,03 & 73,18 & 48,14 & 25,39 \\
\hline 2007 & 40,92 & 26,77 & 14,63 & 27,54 & 7,18 & 21,46 \\
\hline 2006 & 44,75 & 35,54 & 12,20 & 44,82 & 28,53 & 18,90 \\
\hline 2005 & 31,97 & 23,64 & 10,06 & 55,35 & 35,79 & 21,61 \\
\hline Média: & 48,18 & 32,73 & 18,02 & 66,75 & 45,86 & 23,80 \\
\hline
\end{tabular}

Fonte: Cálculos do Autor. 